\title{
Diaphragmatic weakness in hereditary motor and sensory neuropathy
}

\author{
R Hardie, A E Harding, N Hirsch, C Gelder, A D Macrae, P K Thomas
}

\begin{abstract}
Six patients severely affected by hereditary motor and sensory neuropathy (HMSN), four type I and two type II, had clinical evidence of diaphragmatic weakness. One presented with cardiorespiratory failure secondary to nocturnal hypoventilation, and three others were unable to lie flat because of dyspnoea. Diaphragmatic paralysis should be considered as a cause of respiratory or cardiac failure in cases of HMSN.
\end{abstract}

Diaphragmatic weakness is a well recognised cause of nocturnal hypoventilation and respiratory failure in patients with a wide range of neuromuscular disorders, particularly adult onset acid maltase deficiency, motor neuron disease, congenital myopathies, and following childhood poliomyelitis. ${ }^{1-4}$ Its occurrence and consequences in hereditary motor and sensory neuropathy (HMSN) are not well documented. The purpose of this paper is to describe six patients with HMSN and diaphragmatic paralysis to draw attention to this unusual but potentially life threatening complication of a common neurological syndrome.

\section{Case reports}

Case 1 This 67 year old female was admitted for investigation of nocturnal and exertional dyspnoea. It was noted that she walked abnormally and had high arched feet at the age of two years, and in childhood had numerous orthotic devices and surgical procedures for foot deformity. From the age of about 30 years she experienced increasing difficulty in walking and noticed clumsiness and weakness of her hands. Her father and a number of his relatives were similarly affected, as was the patient's brother who died of respiratory failure at the age of 60 years.

The patient smoked heavily until 1986 and had had progressive exertional dyspnoea and wheeziness without cough for 10 years. For three years she had woken frequently at night with acute breathlessness, and slept with four pillows. She had been admitted to hospital on several occasions and had previously been diagnosed as having ischaemic heart disease. She had been unable to lie flat without feeling suffocated for many years and snored loudly during sleep. For one to two years she had been sleepy during the day, had morning headache, and had gained $10 \mathrm{kgs}$ in weight associated with the development of ankle oedema. She was taking captopril and frusemide on admission.

On examination she was obese and cyanosed with signs of congestive cardiac failure. Forced vital capacity (FVC) was $1: 11$ standing and 0.51 lying; the patient became extremely distressed on lying flat and there was striking paradoxical movement of the abdomen when supine, with inward movement during inspiration. There was marked distal wasting and weakness in the limbs with pes cavus and finger contractures. The tendon reflexes were absent and there was distal sensory loss in the legs. The peripheral nerves were thickened.

Electrodiagnostic studies showed undetectable sensory action potentials (SAPs). Distal muscles were inexcitable by peripheral nerve stimulation or magnetic stimulation of the motor cortex. Distal motor latency to flexor carpi ulnaris from the ulnar nerve at the elbow was $16.0 \mathrm{~ms}$. Electromyography (EMG) showed evidence of denervation, more marked in distal muscles; there was no activity in the small hand muscles. ECG showed right axis deviation, and chest radiograph elevation of both hemidiaphragms with poor movement on fluoroscopy. Daytime arterial $\mathrm{pO}_{2}$ was $8.0 \mathrm{kPa}$, $\mathrm{pCO}_{2} 7 \cdot 0 \mathrm{kPa}$, and $\mathrm{pH} 7 \cdot 38$. Sleep studies using continuous finger oximetry showed a baseline saturation of $85 \%$, with four episodes of cyclical dipping to saturations of $40 \%$; each episode lasted about 12 minutes.

Case 2 This 65 year old female was observed to fall frequently at the age of seven years and subsequently had numerous operations for foot deformity. Weakness of the hands was apparent in her twenties, and for 10 years she had used a walking frame or wheelchair. She had been unable to lie flat for many years because of breathlessness.

On examination there were no signs of cardiac failure. She was acutely distressed on lying flat, when FVC was unrecordable; it was 1.51 on standing. There was marked paradoxical abdominal movement with respiration. She had severe distal wasting and weakness, areflexia, distal loss of all sensory modalities, pes cavus, a thoracic kyphosis, and peripheral nerve hypertrophy. SAPs were undetectable and the small hand and foot muscles were denervated. Proximal (supraclavicular fossa- 
elbow) motor nerve conduction velocity (MNCV) was $19 \mathrm{~m} / \mathrm{s}$. The patient had no knowledge of her parents, but one of her daughters was similarly but less severely affected and had electrophysiological evidence of a demyelinating motor and sensory neuropathy.

Case 3 This 35 year old female developed progressive difficulty in walking at the age of seven years, and needed a wheelchair increasingly frequently for about the past 10 years. There was a history of a similar but less severe disorder in her son, father, and paternal grandmother. For one year she had been unable to lie flat because of breathlessness; sleep was frequently interrupted by dyspnoea and she used six pillows.

On examination she was obese and had mild ankle oedema. There was paradoxical abdominal movement and FVC was 1.01 lying, 1.41 standing. The neurological findings were similar to those in case 2 , and electrodiagnostic studies showed evidence of a demyelinating motor and sensory neuropathy. Electrical phrenic nerve stimulation failed to produce any convincing muscle action potentials in the diaphragm. Continuous oximetry did not show significant dèsaturation during sleep. Arterial $\mathrm{pO}_{2}$ was $12.7 \mathrm{kPa}$, and $\mathrm{pCO}_{2} 4.45 \mathrm{kPa}$.

Case 4 This 38 year old female had an abnormal gait at the age of 11 years, and later developed weakness of the hands. Her disability was gradually progressive and she became confined to a wheelchair at the age of 30 . Her father and paternal grandfather were similarly affected. She complained of chronic fatigue and dyspnoea on minimal exertion, but had no symptoms of nocturnal hypoventilation.

On examination she had severe weakness and wasting and loss of all sensory modalities distally in the limbs with areflexia. There was paradoxical abdominal movement on lying flat. FVC was 1.91 standing and 1.61 lying. Electrodiagnostic studies showed evidence of a severe demyelinating peripheral neuropathy. No consistent response was obtained from the diaphragm with phrenic nerve stimulation. Nocturnal continuous oximetry showed no evidence of desaturation during sleep.

Case 5 This 51 year old female was observed to have scoliosis and foot deformity at the age of 11 years and subsequently had progressive difficulty walking and weakness of the hands. She had been confined to a wheelchair for 10 years. She had been breathless on lying flat for many years, sleeping with seven pillows, but had no daytime hypersomnolence or morning headache. Her elder sister was similarly affected but both parents were asymptomatic in their late seventies and there was no consanguinity.

On examination there was mild weakness of neck flexion, moderate distal wasting and weakness in the upper limbs, and more severe generalised weakness, with distal emphasis, in the legs. The tendon reflexes were absent and there was extensive loss of superficial sensation, for pain and temperature more than touch, sparing the face and central abdomen.
Position and vibration sense were reduced distally in the legs. There was paradoxical abdominal movement when she lay flat and FVC was 3.11 standing, 1.61 lying. MNCV in the median and ulnar nerves was 34 and $35 \mathrm{~ms}$ respectively; SAPs were absent. A sural nerve biopsy showed evidence of a chronic axonal neuropathy.

Case 6 This 22 year old female did not walk until the age of two years, and was noted to have footdrop when she was four. Her hands became weak in early childhood, and she had used a wheelchair intermittently since her early teens. Her mother had a similar disorder, was unable to lie flat because of dyspnoea, and died of respiratory problems at the age of 47 years.

On examination there was distal weakness, wasting and sensory loss for all modalities in both upper and lower limbs. The biceps and triceps jerks were present but the other reflexes were absent. There was paradoxical abdominal movement when she was supine. FVC was 2.91 standing and $2 \cdot 11$ lying. Continuous oximetry during sleep showed a baseline saturation of $94 \%$ and two short episodes of desaturation to $88 \%$. No SAPs were detectable. MNCV was 44 $\mathrm{m} / \mathrm{s}$ in the ulnar nerve.

\section{Discussion}

The clinical, neurophysiological and genetic features of four of these patients indicate a diagnosis of autosomal dominant HMSN type I. Cases 5 and 6 had HMSN type II, of autosomal recessive inheritance in case 5 , and autosomal dominant in case $6 . .^{56} \mathrm{~A}$ previous report of diaphragmatic weakness in HMSN type I described a patient who was also diabetic. ${ }^{7}$ Our attention to this association was stimulated by the presentation of case 1 with cardiac failure secondary to nocturnal hypoventilation resulting from diaphragmatic paralysis. This cause of cardiac failure is often overlooked, and should be considered in any patient with neuromuscular disease. Assisted nocturnal ventilation may relieve the secondary cardiac symptoms, improves well being during the day, and can be lifesaving. It may also alleviate diaphragmatic "fatigue", resulting in increased diaphragmatic efficiency during spontaneous daytime respiration. However, case 1 also illustrates the difficulty in managing some patients with neurological disability and diaphragmatic weakness. She could not tolerate a rocking bed; as a result of the weakness of her hands, and living alone, the use of either a cuirass type ventilator or positive pressure ventilation at night was impossible.

Our observations suggest that diaphragmatic involvement may be relatively common in HMSN if the disease is severe or advanced, as all these cases have presented to us in the last year. The patients reported here all had unusually severe disability as a result of HMSN. The neuropathy in HMSN is lengthrelated and, in view of the length of the phrenic nerve, diaphragmatic weakness might not be unexpected. Apart from case 1, only three of the other five patients had prominent symptoms arising from difficulties in breathing when lying flat and it is likely that extreme 
obesity was a contributing factor in case 3 as oxygen saturation did not fall during sleep. It therefore appears that significant morbidity from diaphragmatic paralysis in HMSN is rare, as is suggested by its apparent scarcity in large published series of cases. ${ }^{58}$ Nevertheless, we suggest that diaphragmatic paralysis should be suspected in patients with HMSN who present with symptoms of nocturnal hypoventilation or unexplained cardiac failure, and the diagnosis confirmed by measuring lying and standing FVC, assessment of abdominal movement during respiration in the supine position, and measurement of arterial oxygen saturation during sleep.

We thank Drs J Cassar and J E Rees for referring cases 1 and 5.
1 Newsom Davis J, Goldman M, Loh L, Casson M. Diaphragm function and alveolar hypoventilation. Quart $J$ Med 1976;45:87-100.

2 Trend PSt), Wiles CM, Spencer GT, Morgan-Hughes JA, Lake BD, Patrick AD. Acid maltase deficiency in adults. Diagnosis and management in five cases. Brain 1985;108:845-60.

3 Nightingale S, Bates D, Bateman DE, Hudgson P, Ellis DA Gibson GJ. Enigmatic dyspnoea; an unusual presentation of motor neurone disease. Lancet 1982;i:933-5.

4 Howard RS, Wiles CM, Spencer GT. The late sequelae of poliomyelitis. Quart J Med 1988;251:219-32.

5 Harding AE, Thomas PK. The clinical features of hereditary motor and sensory neuropathy types I and II. Brain 1980;103:259-80.

6 Harding AE, Thomas PK. Autosomal recessive forms of hereditary motor and sensory neuropathy. $J$ Neurol Neurosurg Psychiat 1980;43:669-78.

7 Chan CK, Mohsenin V, Loke J, Virgulto J, Sipski ML, Ferranti R. Diaphragmatic dysfunction in siblings with hereditary motor and sensory neuropathy (CharcotMarie-Tooth disease). Chest 1987;91:567-70.

8 Dyck PJ, Lambert EH. Lower motor and primary sensory neuron diseases with peroneal muscular atrophy. I. Neurologic, genetic and electrophysiological findings in 